brachte Abduktion, Erwärmung Adduktion, Diese Befunde entsprechen denen von Goldste IN. Es wurde Vereisung mit Warmund Kaltspülung des Labyrinths kombiniert. Vereisung auf der Seite der Spülung setzt die Vestibularreaktionen herab, kontralaterale Vereisung steigert sie. Das normale Kleinhirn dürfte eine hemmende Wirkung auf die Labyrinthreflexe ausüben. Es wird eingehend der klinische und anatomische Befund eines Falles mitgeteilt, bei dem ein Drittel der linken Kleinhirnhemisphäre abgetragen wurde. Es wird ein Vergleich mit dem von PötzL und FISCHER mitgeteiltem ersten Fall von paramedianer Kleinhirnresektion durchgeführt. Die Verschiedenheit der Fälle wird nicht allein in der verschiedenen Lage der Resektionsfelder, sondern in der Verschiedenheit der Resektionsrichtung (die beiden Resektionsrichtungen stehen aufeinander normal) zurückgeführt. Die Verschiedenheit $z$ wischen den Symptomen bei lateral-sagittal und paramedian durchgeführter Resektion dürfte in Beziehung stehen zu den beiden normal aufeinander orientierten Fasersystemen der Kleinhirnrinde, die verschieden betroffen sind. STENGEL.

\title{
MAX VON FREY $\dagger$.
}

Am 25. Januar d. J. ist MaX v. Frey, Dr. med. und Dr. phil. h. c., Professor der Physiologie an der Universität Würzburg, aus dem Leben geschieden, achtzigjährig, noch wenige Wochen vor seinem Tode unermüdlich als Forscher und Lehrer seinem Institute vorstehend.

Jede Schönrederei widerspräche der Gesinnung des einzig artigen Mannes und dem Schüler, der nur in herzlicher Verehrung seiner gedenken kann, erscheinen alle Worte zu abgegriffen, um damit dem Wesen des Toten gerecht werden zu können. Die kurze Darstellung seines Lebens und seiner Arbeit, um welche die Schriftleitung dieser Wochenschrift den Verfasser ersuchte, soll dazu beitragen, den Verlust, den seine Freunde und Schüler und darüber hinaus die Physiologie und Medizin durch sein Scheiden erlitten hat, recht zu beleuchten.

Am I6. November 1852 in Salzburg geboren, studierte M. v. FREY in Wien, Leipzig, Freiburg i. B. und München Medizin. Schon als Student trat er in den Mitarbeiterkreis des großen Leipziger Physiologen LUDWIG ein und vollendete seine erste Experimentalarbeit über die Innervation der Speicheldrüsen. Nach Abschluß seines Studiums kehrte er im Jahre r88o als Assistent in das Ludwigsche Laboratorium zurück und erlebte in dieser Stellung den Tod seines großen Lehrers. Nach zwei weiteren Dozentenjahren unter der Leitung E. Herings erfolgte 1897 seine Berufung nach Zürich, von wo aus er im Jahre I899 als Nachfolger Adolf Ficks nach Würzburg gewählt wurde.

In welchem Maße er die große Tradition des Würzburger Instituts zu wahren wußte, welche Intensität der Forschung und de Anleitung seiner Schüler er in seiner Würzburger Arbeitsperiode entfaltete, darüber berichten die 30 Bände „Arbeiten aus dem Physiologischen Laboratorium der Würzburger Hochschule", die er der wissenschaftlichen Welt schenken konnte.

Abgesehen von den zahlreichen Arbeiten aus dem Gesamt gebiete der Medizin und Physiologie, die unter seiner Leitung entstanden, für welche er - und das war charakteristisch für ihn stets die volle Autorschaft dem Bearbeiter übertrug, sind dre große Forschungsperioden aus seinen eigenen Schriften herauszufinden, deren Ergebnisse von fundamentaler Bedeutung wurden. Die erste war der Muskelphysiologie, die zweite der Physiologie des Blutkreislaufes und die letzte der Physiologie der Hautsinne gewidmet.

Die Einsicht in die Physiologie der Muskulatur erhellte er durch seine Untersuchungen über den Gaswechsel des Skeletmuskels sowie seine Experimente zur Muskelmechanik. Die Diskontinuität der tetanischen Erregung stellte er in eleganten Versuchen sicher und intensiv beschäftigte ihn das allgemeine Problem des Kontraktionsvorganges. Das Ergebnis aller dieser Arbeiten und Überlegungen fand einen Niederschlag in dem durch seine Klarheit unverändert vorbildlichen Abschnitt über ,Allgemeine Physiologie der quergestreiften Muskeln" des Nagelschen Handbuches der Physiologie.

Die Verdienste M. v. Freys um die Ablösung rein qualitativ beobachtender Methoden durch exakt messende, unverzerrt registrierende Verfahrungsweisen unter Ausnützung der Handhaben, welche die Physik bot, kamen außer in seinen Muskelexperimenten hauptsächlich zum Ausdruck in seinen Kreislaufuntersuchungen. Eine abschließende Monographie "Über den Puls" war seinem Lehrer Ludwig gewidmet. Sie ist auch heute noch eine der beachtenswertesten und vollkommensten Darstellungen dieses Gebietes.

Am geläufigsten ist die Verknüpfung seines Namens mit der Physiologie der Hautsinne. Wenn man heute in der Lage ist, den Tastsinn der Körperoberfläche in den einzelnen Qualitäten des Druck-, Schmerz-, Wärme- und Kältesinnes unter Zuordnung jeweils spezifischer Receptoren, Nervenbahnen, Reizbedingungen usw. klar zu durchschauen, so ist das fraglos in erster Linie das große Verdienst der unermüdlichen und scharfsinnigen Experimentierarbeit M. v. Freys. Die Grenzfläche zwischen Umwelt und Organismus und die Möglichkeit der Einwirkung von Umweltkräften auf nervösem Wege auf den Organismus war für ihn das große Problem. Neben rein sinnesphysiologischen Interessen leiteten ihn dabei vor allem stets allgemeinphysiologische Gesichtspunkte. Im Zusammenhange mit diesem Forschungsgebiet beschäftigte ihn auch die Frage der sensiblen Muskelreceptoren, welche der Spannungs- und Kraftempfindung dienen, sowie die Frage der "Tiefensensibilität", wobei er die Bedeutung des Hautdrucksinnes für die Erkennung von Gliederstellung und Gliederbewegung entdeckte. Die Wichtigkeit dieser seiner letzten Arbeitsepoche für die Medizin wird sich erst voll äußern, wenn die Klinik sich dazu entschließen wird, Ernst zu machen mit der exakten quantitativen Untersuchung der peripheren Receptoren und ihrer Erregungsbedingungen, was M. v. FREY ihr in mehreren Referaten und Kongreßberichten des öfteren nahegelegt hat.

Prunkvolle Darstellungen, aufsehenerregende Theorien verabscheute v. FREY ebenso wie ",vorläufige Mitteilungen". Wohltuend nüchtern und schlicht ist die Art seiner wissenschaftlichen Abhandlung. Auch die glanzvoll gesetzte gefällige Rede lag $\mathrm{ihm}$ nicht. Darauf beruhte die Eigenart seines Unterrichtes. Aus Experimentaldaten oder gebotenen Beobachtungen entwickelte er die Zusammenhänge oder überließ deren Entwicklung dem Lernenden selbst. Er stellte Anforderungen an seine Hörer und war weit davon entfernt, dem Interesselosen „Examenskenntnisse" $z u$ vermitteln, entsprechend seiner hohen Auffassung von den Aufgaben des deutschen Hochschullehrers. Das von ihm geleitete und hervorragend entwickelte "Physiologische Praktikum" stand, wie danach verständlich ist, ganz im Mittelpunkt seiner Unterrichtstätigkeit. Bezeichnend für seine Lehrart sind seine ,Vorlesungen über Physiologie". Ohne jeden Umschweif, ohne ein Wort zuviel, bietet dieses Buch von höchster Warte eine Fülle kritisch gesichteten Wissens und darf unter die vorzüglichsten Lehrbücher der physiologischen Weltliteratur gerechnet werden.

M. v. FREY war ein Meister des Experiments auf allen Gebieten seines Faches. Wer das Glück hatte, als Mitarbeiter in seinem Laboratorium weilen zu dürfen, der konnte vor allem die ,Disziplin des Experiments" erlernen wie an kaum einem Ort. Nichts war v. FREY unverständlicher als ein methodisch unvorbereiteter Versuch oder gar ein Experiment ohne klare Fragestellung. Er hielt nichts, wie in einer seiner Schriften zu lesen ist, vom Vorwärtskommen ",mit Siebenmeilenstifeln“, sondern empfahl, ,unermüdlich Hypothesen zu spinnen und diese durch gewissenhafte Experimente und nüchterne Beobachtungen nachzuprüfen". Bewundernswert war seine stete Orientierung nicht nur in seinem engeren Fache, sondern vor allem auch in den praktischen und theoretischen Grenzgebieten bis in die letzten Wochen seines Lebens. Nie klopfte man vergebens an seine Tür. Stets war er auch für den jüngsten seiner Institutsmitglieder ein gütiger und hilfsbereiter Berater.

Das Leben v. FreYs erschöpfte sich keineswegs im Laboratorium und in der Studierstube. Seine einzigartige Weltklughei erklärte sich durch sein Aufgeschlossensein für alle Ereignisse und Fortschritte menschlichen Lebens. Noch mit 75 Jahren konnte man ihn beispielsweise auf dem Flugplatz der Würzburger Fliegerschule die Maschine besteigen sehen, bestrebt, đie Möglichkeiten des Menschenfluges aus eigener Erfahrung kennenzulernen. Seine noch in den letzten Jahren ausgeführte Reise zu Gastvorlesungen in den Vereinigten Staaten war, wie er versicherte, nicht eine Strapaze, sondern ,,voller Anregungen“. Seine beispiellose Selbstbeherrschung, seine umfassende wahre Bildung in jeder Hinsicht ließen ihn jeder Situation gewachsen erscheinen. Seine Herzensgüte äußerte sich in schlichter Liebenswürdigkeit, durch die er sich rasch die Zuneigung aller derer erwarb, die mit ihm in Berührung kamen

In aller Stille ist der große Mann dahingegangen, von seinen Schülern geliebt und verehrt als Vorbild eines Menschen und Forschers, als nie versagender Helfer und Berater inmitten einer nervös hastenden, sensationslüsternen Zeit, deren Lärm bis in die Werkstätten der Wissenschaft dringt und Menschen seines Schlages seltener und seltener werden läßt.

H. Rein, Freiburg i. Br. 